\title{
THE STELLAR POPULATION OF THE M31 SPIRAL ARM AROUND OB ASSOCIATION A24
}

Keiichi Kodaira ${ }^{1}$, Vladas Vansevičius ${ }^{1,2}$, Motohide Tamura $^{1}$, and Satoshi Miyazaki ${ }^{1}$

Received 1998 October 28; accepted 1999 February 2

\footnotetext{
${ }^{1}$ National Astronomical Observatory, 2-21-1 Osawa, Mitaka, Tokyo 181-8588, Japan; kodaira@cc.nao.ac.jp, vladasvn@cc.nao.ac.jp, tamuramt@cc.nao.ac.jp, satoshi@merope.mtk.nao.ac.jp

${ }^{2}$ Institute of Physics, Goštauto 12, Vilnius 2600, Lithuania; wladas@itpa.lt
} 


\begin{abstract}
A study of the stellar population of the M31 spiral arm around OB association A24 was carried out based on the photometric data obtained from deep $V$ and $J H K$ imaging. The luminosity function was obtained for $-7 \lesssim M_{b o l} \lesssim-3.5$ by applying the extinction correction corresponding to $A_{V}=1$ and the bolometric correction $B C_{K}$ as an empirical function of $(J-K)_{0}$. In comparing the observed color-luminosity diagrams with semitheoretical isochrones modified for the dust-shell effects, we found the young population of $t \lesssim 30$ Myr with supergiants of $M_{b o l} \lesssim-5$, the bulk of the intermediate-age population of $t \sim 0.2-2.5$ Gyr with bright asymptotic giant branch (AGB) stars of $-5 \lesssim M_{b o l} \lesssim-4$, and old populations of $t \gtrsim 3$ Gyr with AGB and red giant branch (RGB) stars of $M_{b o l} \gtrsim-4$. The average star formation rate was estimated to be $\sim 1.8 \times 10^{4} m_{\odot} \mathrm{Myr}^{-1}$ and $\sim 0.7 \times 10^{4} m_{\odot} \mathrm{Myr}^{-1}$ per deprojected disk area of $1 \mathrm{kpc}^{2}$ from the number density of B0 V stars around $M_{V}=-4.0$ (age $\sim 10 \mathrm{Myr}$ ) and the number density of bright AGB stars around $M_{\text {bol }}=-4.3$ (age $\sim 1 \mathrm{Gyr}$ ), respectively. A study of the local variation in the $V$ and the $J$ and $H$ luminosity functions revealed a kind of anticorrelation between the population of the young component and that of the intermediate-age component when subdomains of $\sim 100$ pc scales were concerned. This finding suggests that the disk domain around the A24 area experienced a series of star formation episodes alternatively among different subdomains with a timescale of a few spiral passage periods. Brief discussions are given about the interstellar extinction and about the lifetimes of bright AGB stars and the highly red objects (HROs) in the same area.
\end{abstract}

Subject headings: galaxies : individual (M31) — galaxies : photometry — 
galaxies : star clusters — galaxies : stellar content — infrared : galaxies — stars : AGB and post-AGB 


\section{Introduction}

The Andromeda galaxy (M31) is the nearest galaxy which has a morphological type close to that of the Milky Way, and is most suitable to provide supplementary data for understanding the detailed galactic structures such as spiral arms and related star forming regions. With this motivation in mind, the present authors carried out deep near-infrared stellar photometry around the OB association A24 (R.A. $=0^{h} 39^{m} 18^{s}$, Decl. $=+40^{\circ} 41^{\prime}[1950.0]$ ) near the $7 \mathrm{kpc}$ spiral arm of M31 (see Fig. 1), and published the basic photometric data separately (Kodaira et al. 1998a, hereafter Paper I). In the present paper we carry out a population study of the stars around A24 based upon the near-infrared data in Paper I and the new $V$-band data.

An automated search for OB associations in M31 was carried out by Magnier et al. (1993) based upon the CCD survey data of Magnier et al. (1992), and Haiman et al. (1994) analyzed the properties of a dozen OB associations selected from the list of Magnier et al. (1993). In order to improve the data quality, Magnier et al. (1997) undertook $U B V$ photometry using the Hubble Space Telescope (HST) WFPC for 15 fields related to the OB associations A41, A42, and A48. They found that the median ages of the bright blue stellar components were $\log t$ (years) $=7.0-7.5$ with an average $\log t \sim 7.3$, and that the total interstellar extinction of individual stars was highly variable over a range $A_{V}=0-3$ with group median values of $A_{V} \simeq 0.6-1.3$ and an average of $A_{V} \sim 0.9$ assuming $R_{V}=3.1$.

The present target, OB association A24 (area $\sim 2^{\prime} \times 1 .^{\prime} 5$ ), is composed of a scattered group of moderately faint stars, with a core size of about $0.1 \mathrm{kpc}$ (van den Bergh 1964; see Fig. 1). This core includes open clusters C202 and C203, both small with bright stars, near the rim of the dark cloud D221 (area $\sim 0 .^{\prime} 8 \times 0 .{ }^{\prime} 8$ ), which appears at the central part of A24 (Hodge 1981). Efremov, Ivanov, \& Nikolov (1987) identified the original A24 as A24-1, and additionally defined new associations A24-2 and A24-3 on the southeast outside 
A24-1. They noticed that A24-1 was a stellar complex which contained at least five internal groupings. Other dark clouds, D205, D215, and D253, and a faint open cluster C204 are seen in the vicinity of A24.

The clouds D221 and D253 compose a part of the spiral dark lane which appears to branch off inward from the $10 \mathrm{kpc}$ main dark lane on the south side of M31. The HI distribution maps by Unwin (1980) and Brinks \& Shane (1984) show that an HI spiral arm runs through D221, but that the association A24 including the cloud D221 is located at a local minimum of HI distribution along the spiral arm. The IRAS maps for the emission at 60 and $100 \mu m$ by Xu \& Helou (1996a) also show that the spiral arm is weakening in the vicinity of A24. Clear signs of diffuse $\mathrm{H} \alpha$ emission were seen in the A24 area in the $\mathrm{H} \alpha$ survey maps of Pellet et al. (1978) and of Devereux et al. (1994). The $\mathrm{H} \alpha$ features were more clearly identified by Magnier et al. (1995) in their search for supernova remnants (SNRs) in M31. Their study revealed that a superbubble of $38^{\prime \prime}(\sim 130 \mathrm{pc})$ diameter developed around the open clusters C202 and C203, with two possible SNRs in the neighborhood.

These features of A24 (with a superbubble and at a local minimum of the HI spiral arm) highly resemble to those of A41a, which was investigated by Magnier et al. (1997). They found A41a to have a median age of $\log t \sim 7.3$ and a median extinction of $A_{V} \sim 0.6$. The foreground reddening in the Milky Way toward M31 was estimated at $E_{f}(B-V)=0.16$ (Sandage \& Tammann 1968), $E_{f}(B-V)=0.07$ (Humphreys 1979), or $E_{f}(B-V)=0.08$ (Burstein \& Heiles 1984). Hodge \& Lee (1988) found internal reddening $E_{i}(B-V)=0.21$ for A24. Our reanalyses of the blue stars in the Hodge \& Lee (1988) field using their $U B V$ data and the $B V R I$ data of Magnier et al. (1992) resulted in $E_{i}(B-V) \simeq 0-1$ for individual stars, with a median value of $E_{i}(B-V)=0.23$. The extinction seems to be highly inhomogeneous over the A24 field, as was found by Magnier et al. (1997) for other 
associations.

Since we are mainly dealing with the near-infrared data, the uncertainty and the inhomogeneity of the extinction little affect the interpretation about the global characteristics of the stellar population. Accordingly, in the present population study we assume an average extinction of $A_{V}=1.0$, which corresponds to $A_{K}=0.11$, $E(J-K)=0.17, E(H-K)=0.07$, and $E(B-V)=0.32$ when the extinction law for $R_{V}=3.1$ is adopted (Mathis \& Cardelli 1990). The main conclusions from the present study are hardly affected by an uncertainty of $\Delta A_{V}= \pm 0.2$. Since the photometric zero point of the $K$ magnitude was adjusted in Paper I by fitting the observed main sequence to the unreddened one in the $(J-H)$ versus $(H-K)$ diagram, we need in the case of $A_{V}=1.0$ to subtract corrections $\Delta(J-H)=0.10, \Delta(J-K)=0.10$, and $\Delta K=0.07$ from the photometric data given in the Paper I, for the comparison with (unreddened) theoretical values. The colors with subscript zero and the absolute magnitudes are for the dereddened values, while the original data in the Paper I photometric system appear without a subscript zero in the present paper.

In the following, a description of the observational material is given in $\S 2$, and a population study is presented in $\S 3$. We will investigate the local variations of stellar population and of interstellar extinction in $\S 4$. Discussions of star formation and cloud structure in the A24 area are given in $\S 5$. Brief discussions are also given in $\S 5$ of the lifetimes of bright AGB stars and of the HROs identified by Kodaira et al. (1998b).

\section{Observational Material}

Our near-infrared data consist of two sets, which were both obtained with the QUIRC imager attached to the University of Hawaii $2.2 \mathrm{~m}$ telescope at Mauna Kea. One set is the 
$J H K$ data obtained in 1995 September with the f/31 tip-tilt secondary mirror, and their point-spread function $(\mathrm{PSF})$ is as good as $\mathrm{FWHM} \simeq 0 . " 4$. This set of high-quality data covers the central field of $2^{\prime} \times 2^{\prime}$ around D221 and reaches down to $J=21.3, H=20.5$, and $K=19.6$. The other set is the $J H$ data, which were obtained in 1996 December with the f/10 normal secondary mirror and covered a $3^{\prime} \times 3^{\prime}$ field around the f/31 field. The set of the $\mathrm{f} / 10 J H$ data has a PSF of FWHM $\simeq 0 .{ }^{\prime \prime} 9$ and reaches down to $J=20.0$ and $H=19.0$. More details about the near-infrared photometry are to be found in Paper I.

The $V$-band image was obtained on 1995 September 23 using the University of Hawaii 8K mosaicked CCD camera at the $\mathrm{f} / 3.8$ primary focus of the Canada-France-Hawaii Telescope $(\mathrm{CFHT})$. The mosaic of eight $2 \mathrm{~K} \times 4 \mathrm{~K}$ front-illuminated detectors with $15 \mu \mathrm{m}$ pixels $\left(0 .{ }^{\prime \prime} 23\right.$ pixel $\left.^{-1}\right)$ covered a $0 .{ }^{\circ} 52 \times 0 .{ }^{\circ} 52$ field of the south part of M31 including the spiral arms around A24. The $900 \mathrm{~s}$ exposure frame was debiased using a $900 \mathrm{~s}$ dark frame and flattened using the sky flat field, which was produced by taking a median of 20 frames. The final object frame has stellar images of FWHM $\sim 0 . " 9$. A $7^{\prime} \times 7^{\prime}$ field around A24 was carefully measured to yield a $V$-band magnitude for about 29,000 stars down to $V \sim 24$. The photometric calibration was made by referring to the M31 survey data by Magnier et al. (1992). The resulting $V$-band luminosity function is given in Figure 2, which shows 3 components corresponding to supergiants $\left(M_{V} \lesssim-6\right)$, OB main-sequence stars $\left(-6 \lesssim M_{V} \lesssim-3.5\right)$, and RGB and AGB stars $\left(-3.5 \lesssim M_{V}\right)$.

We will use the high-quality $\mathrm{f} / 31 J H K$ data for the population study for the $2^{\prime} \times 2^{\prime}$ central field, and the $\mathrm{f} / 10 \mathrm{JH}$ data and the $\mathrm{f} / 3.8 \mathrm{~V}$ data for the study of local variation in the stellar population and the interstellar extinction over the $3^{\prime} \times 3^{\prime}$ and the $7^{\prime} \times 7^{\prime}$ fields, respectively. 


\section{Population Study}

We begin with the population study of the 3139 stars observed in the $J H K$ bands in the $2^{\prime} \times 2^{\prime}$ central field of A24 (see Fig. 1b), whose effective area is $\sim 3$ arcmin $^{2}$ because of mosaicking seams. The $K$-band luminosity, $M_{K}$, was transferred to the bolometric luminosity, $M_{b o l}$, using the empirical relation between $(J-K)_{0}$ and the $K$-band bolometric correction $B C_{K}$, which were derived by us based upon the data in Whitelock et al. (1995), Kučinskas (1998), and Montegriffo et al. (1998). The details of the transformation are given in Appendix A.

The conventional value of the distance modulus $m-M=24.2$ (Welch et al. 1986; Pritchet \& van den Bergh 1987; Huterer, Sasselov, \& Schechter 1995) is adopted for M31 throughout this paper, although Stanek \& Garnavich (1998) and Holland (1998) are proposing a larger value, $m-M=24.47$, based upon HST $V I$-band data about the "red clump" stars and the RGB stars, respectively. Our main conclusions in the following are hardly affected by the ambiguity of $\Delta(m-M)=+0.3$. The resulting luminosity function is shown in Figure 3. The luminosity function shows three components that correspond to the few brightest objects with $M_{\text {bol }} \lesssim-6.5$, bright stars with $-6.5 \lesssim M_{b o l} \lesssim-5$, and the dominant component with $M_{b o l} \gtrsim-5$. The brightest part of this luminosity function for $M_{b o l} \lesssim-6$ is subject to large statistical uncertainty because of the scarceness of these stars in the $2^{\prime} \times 2^{\prime}$ field. The change of slope that is seen in the $K$ magnitude distribution around $K \sim 17.5$ (see Fig. 4b in Paper I) is not clear in Figure 3.

In Figures 4 and 5 we compare the observed two-color diagram (TCD) and colormagnitude diagrams (CMDs) with the isochrones, of which the original theoretical ones are based on those of the Padova group (Bertelli et al. 1994, hereafter Padova 94). We have plotted only 844 stars of $K<18.0$ in Figure 4 because the colors of the fainter stars are increasingly subject to the photometric errors (see Paper I). In the TCD the Padova 
94 isochrones for various ages and metallicities are almost degenerated, and cannot cover the upper domain where the observed red stars of $J-H>0.7$ are distributed, while the tracks for RGB and AGB stars along $J-K \simeq 1.2$ extend far beyond the populated domain. This discrepancy between the observed stellar distribution and the Padova 94 isochrones in TCD may mainly be attributed to the following two causes. One apparent cause is connected to the difficulties in transforming the theoretical HR diagram $(\log L$, $\left.\log T_{\text {eff }}\right)$ into the color-magnitude diagram. Because of the lack of a sufficient spectral library for evolved cool stars and the uncertainty in the correspondence between the spectral types and the theoretical parameters $\left(g, T_{\text {eff }}\right)$ for these stars, Padova 94 had to invoke various simplifications in producing spectral energy distributions (SEDs) for stars of low effective temperature $\left(T_{\text {eff }}<3500 \mathrm{~K}\right)$ and low surface gravity $(\log g<3)$. The second cause is neglect of the effects of the dust shells which are probably ejected during the AGB evolution, which was not taken into account in the Padova 94 models. Bressan, Granato, \& Silva (1998) modeled the effects of the dust shells and reproduced fairly well the observed color characteristics of the IRAS AGB stars in the South Galactic Cap (Whitelock et al. 1995). In doing so, they adopted the underlying photospheric radiation roughly corresponding to those halfway on the Padova 94 evolutionary tracks of AGB stars with colors $(J-H)_{0} \simeq 0.9-0.7$ and $(H-K)_{0} \simeq 0.2-0.5$, and modified them for the dust-shell effects. If we apply the dust-shell corrections to the photospheric radiation corresponding to the latest stage of the Padova 94 AGB evolution, which has almost the constant colors of $(J-H)_{0} \simeq 0.5$ and $(H-K)_{0} \simeq 0.8$, the resulting colors do not match the observed ones; they are too red in particular in $(H-K)_{0}$. The deviation of the original Padova 94 isochrones from the observed stellar distribution in CMDs (see Fig. 5) also indicates the necessity of modifying the colors of the evolved cool stars. In extrapolating SEDs along with varying colors $(V-K)$ and $(J-K)$ in Padova 94, there might have been some anomalous behaviors in the $H$ band which led to the unexpected extent of the tracks 
for AGB and RGB stars in TCD, namely, the $H$-band flux might have become too small.

The supplement to the SED library and/or the improvement of the correspondence between the spectral types and the physical parameters are outside the scope of the present observational work. Here we adopt the practical way to empirically terminate the color evolution of AGB stars halfway along the Padova 94 tracks and to modify the colors by correcting them for the dust-shell effects. For the present purpose we adopted $(J-H)_{0}=0.7$ and $(H-K)_{0}=0.3$ as the average terminal colors for the AGB evolution (but not for the RGB evolution) and applied the dust-shell corrections to them. The dust-shell corrections normally become effective for high-luminosity stars of $M_{b o l} \lesssim-3.8$. The details of the dust-shell models are described in Appendix B. Note that the present dust-shell model assumes the silicate grains, which have different extinction properties from those for the standard Galactic interstellar absorption, and that the combined effects of the silicate absorption and the thermal emission from the silicate dust-shell together mimic the standard Galactic reddening in Figure 4. The main isochrones in Figures 4 and 5 are the semitheoretical ones which were derived this way, and the original Padova 94 isochrones are shown in the insets for comparison. We have adopted the isochrones for the helium and metal fractions $Y=0.25$ and $Z=0.008$ for the ages of 5 and $10 \mathrm{Gyr}$, and $Y=0.28$ and $Z=0.02$ for younger ages. The modified isochrones are still partially degenerated in TCD, and the tracks for the RGB stars remain the same, for which no modifications have been applied. These semitheoretical isochrones in CMDs are useful to lend us a guiding reference, while the present population study of AGB stars relies on $M_{b o l}$ rather than on the detailed colors in their late evolutionary phase.

By investigating the CMDs, we find that the youngest population has an age of $t \lesssim 30 \mathrm{Myr}$ when a metallicity similar to that of the solar value, $Z \sim 0.02$, is adopted. If $Z \sim 0.05$ is adopted, the age becomes slightly younger. The brightest stars in the open 
clusters C202 and C203 (the two most luminous) must be as massive as $m \gtrsim 60 m_{\odot}$ and as young as $t \lesssim 4$ Myr if measured images are actually resolved single stars. Note that the youngest main-sequence stars of $M_{b o l} \gtrsim-5.5$ are below the detection limit in the $K$ band. Judging from the broad ridge running through the bright AGB stars of $M_{b o l} \sim-5$ down to $M_{b o l} \sim-3.5$ in CMDs, we find the ages of the bulk of the bright AGB population $\left(-5 \lesssim M_{b o l} \lesssim-4\right)$ to be $t \sim 0.2-2.5$ Gyr for $Z=0.02$, as normally expected for stars of about $\sim 1.5-4 m_{\odot}$. The component of $M_{b o l} \gtrsim-4$ includes the old population of $t \sim 3-10$ Gyr having $Z<0.02$. We suspect that the core part of the lower right extent of the stellar distribution in CMDs (Fig. 5) may include RGB and AGB stars of $M_{b o l} \gtrsim-3.0$ which are suffering from the local absorption of $A_{V} \sim 1-3$ in M31 in addition to the average $A_{V}=1$ adopted above. The reddening effects for these faint stars, however, are not distinct in TCD because the size of the observational errors rapidly increases with decreasing stellar brightness toward the detection limits (see Paper I). For reference we have indicated in Figure 5 a a dereddening vector for $A_{V}=5$ according to the Galactic reddening law.

The observed bright AGB stars of $M_{b o l} \lesssim-4$ are found to be distributed in the domain of the Mira variables in TCD (Bessell \& Brett 1988), corresponding to the redder group of the South Galactic Cap IRAS AGB stars (Whitelock et al. 1995). The majority of the observed objects of $-4<M_{b o l}<-3$ shows concentration in the domain of the semiregular variables in TCD (Kerschbaum, Lazaro, \& Habison 1996).

The possible contamination by the foreground Galactic stars is expected to be about 3 in a $1^{\prime} \times 1^{\prime}$ observed field for $K \leq 19$ (see Rich, Mould, \& Graham 1993) and can be neglected in the present statistical analysis. On the contrary, judging from Kent's (1989) model and near-infrared surface photometry by Hiromoto et al. (1983), Battaner et al. (1986), and Martinez Roger, Phillips, \& Sanchez Magro (1986), the contamination by the stars in the outer bulge of M31 may not be negligible because of the inclination angle of 
M31 at $i=12 .{ }^{\circ} 5$. The bulge stars, however, are most probably old enough to be $t>3 \mathrm{Gyr}$, and the present discussions about A24 focused on the stars of $M_{b o l} \lesssim-4$ are hardly affected by their contamination.

\section{Local Variation of Stellar Population}

In order to study possible local variation of the stellar population around A24, we have derived the magnitude distributions for individual $20^{\prime \prime} \times 20^{\prime \prime}$ mesh fields in the $7^{\prime} \times 7^{\prime}$ $V$-band field as well as in the $3^{\prime} \times 3^{\prime} J$ - and $H$-band fields (Figs. 6 and 9). Using the number density of stars for $22 \leq V \leq 22.5$ as a criterion, we rather arbitrarily identify 33 subdomains in the $7^{\prime} \times 7^{\prime}$ field by combining mesh fields of similar stellar density. Each of such subdomains has an area of about $0.8 \mathrm{arcmin}^{2}$, and they are numbered from 1 to 33, see Figure 6. There remain mesh fields that are not combined into subdomains because of relatively high local fluctuation of stellar density. By analyzing properties of the $V$-band luminosity functions of these subdomains, we find that the main features of the luminosity function can be reproduced by synthesizing a young bright component, $\psi_{1}(V)$, and an older faint component, $\psi_{2}(V)$, with a varying ratio of $r_{V}=\psi_{1} / \psi_{2}$, say at $V=22$. The supergiant component seen in Figure $2\left(M_{V} \lesssim-6.0\right)$ is too scarce in each subdomain and is ignored in defining $\psi_{1}$. The value of $r_{V}$ ranges from 0.1 to 1.1, and some of the typical cases are shown in Figure 7. Both $\psi_{1}(V)$ and $\psi_{2}(V)$ can be approximated in Figures 2 and 7 by a power law, $\log \psi_{i}=A_{i} V+B_{i}$, with $A_{1}$ and $A_{2}$ being $\sim 0.50$ and $\sim 2.24$, respectively. The level constant $B_{2}$ for the older faint component $\psi_{2}(V)$ seems to decrease systematically outward reflecting the density gradient in the M31 disk: $B_{2}($ No.14 $) \simeq B_{2}($ No.32 $) \simeq B_{2}($ No.1 $) \times 0.7$, and $B_{2}(N o .22) \simeq B_{2}(N o .30) \times 0.95$.

In addition to this main property, many subdomains reveal a sign of interstellar extinction, that is, a horizontal shift of the luminosity function toward the fainter side; 
$\psi_{2}(V)=\psi_{2,0}(V-\Delta V)$ with the subscript zero being for the least absorbed, reference subdomain of similar population characteristics. The value of $\Delta V$ ranges from 0 to 0.5 , and the typical cases are shown in Figure 8. We classify the local stellar population by the apparent extinction $\Delta V$ and the ratio of the young to the old component $r_{V}$. The resulting variations in $r_{V}$ and $\Delta V$ are indicated in Figure 6 by shading and asterisks. It is seen that the high- $r_{V}$ subdomains compose the spiral arm.

The distribution of high- $\Delta V$ subdomains matches well with the visual impression of the $V$-band image (Fig. 1a). The relation between $\Delta V$ and the actual extinction $A_{V}$, however, is complicated, for the absorbing matter is mixed with stars in the M31 disk. If we apply a simple model in which the absorption layer is geometrically thin and lies just halfway along the line of sight, $\Delta V \sim 0.15$ may mean complete opaqueness of the layer. Actual absorbing clouds seem to be highly inhomogeneous, and the average opaqueness derived from the shift of the luminosity function may strongly reflect the optically thin parts. In this sense, $\Delta V \sim 0.15$ may also be interpreted by a model in which a fraction 0.5 of the field coverage is fully opaque and the other 0.5 is completely transparent. Xu \& Helou (1996b) evaluated the average extinction at $A_{V} \simeq 1$ from the observed far-infrared flux around $R=7 \mathrm{kpc}$, integrated along the line of sight. Therefore, when inhomogeneities are taken into account, a significant fraction of the field of view may effectively have a fully opaque layer.

We similarly introduce 15 subdomains in the $3^{\prime} \times 3^{\prime}$ field by combining mesh fields according to the number of stars of $J=18-19$ in each mesh field. Each such subdomain has an area of about $0.6 \operatorname{arcmin}^{2}$, and the subdomains are numbered from 1 to 15; see Figure 9. There again remain mesh fields which are not combined into subdomains because of relatively high local fluctuation. The $J$ - and $H$-band luminosity functions (Fig. 10) for the f/10 field show variations from one subdomain to another in the ratio of the population for $H \sim 17.6$ relative to that for $H \sim 18.2, r_{H} \equiv \psi_{H}(17.6) / \psi_{H}(18.2)$. The subdomains in 
Figure 9 are shaded according to the value of $r_{H}$. Judging from the luminosity function for the $H$ band of the $\mathrm{f} / 31$ data, which reaches down to $H=20.5$ (see Fig. $4 \mathrm{~b}$ in Paper I), these two magnitude ranges apparently reflect the intermediate-age population $(t \sim 1$ Gyr) and the older ones ( $t \sim 10 \mathrm{Gyr}$ ), respectively. Note, however, that this part of the distribution functions of the $\mathrm{f} / 10$ data is already subject to the selection effects due to the observational limit. We additionally find a kind of anticorrelation between the intermediate-age population and the young population $(t<100 \mathrm{Myr} ; H<16.5)$ among the subdomains. The same characteristics are also found in the $J$-band luminosity function, as shown in Figure 10a. It is most interesting to note that the subdomains rich in the young population have less intermediate-age population compared with the subdomains that are poor in the young population. No clear sign of differential extinction can be confirmed in the study of the $J$ - or $H$-band luminosity function for the subdomains. The subdomains of different characteristics are indicated in Figure 9 according to the value of $r_{H}$. The global trend of the distribution of the subdomains rich in the young population coincides between the $V$ study and the $J H$ study for the common $3^{\prime} \times 3^{\prime}$ field.

\section{Discussion}

The present bolometric luminosity function (Fig. 3) well resembles that in the M31 "disk" which was derived by Rich et al. (1993) in their Field 2 at 11.'5 off center along the southeast minor axis, except for the brightest end of $M_{b o l}<-6$, which reflects the additional youngest population in A24. The slope is roughly $\Delta \log \psi / \Delta M_{b o l} \sim 1$ for $-3.5<M_{b o l}<-6$ in both studies, although we have noted the substructures in our case in Figure 3 .

In applying the fuel consumption theorem of Renzini \& Buzzoni (1986) for a single stellar population, $n_{j}=B(t) L_{T} t_{j}$, where $n_{j}$ is the number of stars in post-main-sequence 
evolutionary phase $j, t_{j}$ is the lifetime of the phase, $L_{T}$ is the total bolometric luminosity of the population, and $B(t)=2 \times 10^{-11}$ stars $L_{\odot}^{-1} \mathrm{yr}^{-1}$, we may examine the standard lifetime estimate of $1.3 \mathrm{Myr}_{\mathrm{mag}^{-1}}$ for AGB stars (Iben \& Renzini 1983). We use $\mu_{K} \sim 16.5$ mag $\operatorname{arcsec}^{-2}$ for the M31 disk at $R \sim 7 \mathrm{kpc}$ according to Battaner et al. (1986) and $B C_{K} \sim 3.0$, to derive $L_{T} \sim 10^{6} L_{\odot}$ for the whole $\mathrm{f} / 312^{\prime} \times 2^{\prime}$ field (effective area $\sim 10,800$ $\left.\operatorname{arcsec}^{2}\right)$. Although the population of the AGB stars of $-5 \lesssim M_{b o l} \lesssim-4$ are not of single age, we may apply the above equation to the sum of the stars of intermediate ages by virtue of its linearity in $L$ and $n$ when $t_{j} \mathrm{mag}^{-1}$ is constant. For stars of $M_{b o l}=-4.0$ and $M_{b o l}=-5.0$, we find $n_{j} \sim 400$ and $n_{j} \sim 50$, therefore, estimates of $\log t_{j} \sim 6.6$ and $\log t_{j} \sim 5.7$, respectively. The simple average $\log t_{j} \sim 6.15$ is close to the standard value cited above $\left(\log t_{j}=6.1\right)$, basically conforming to the fuel consumption theorem.

When we apply the same fuel consumption theorem for evaluating the lifetime of the luminous HROs of $J-K \gtrsim 2$, which were identified in the $\mathrm{f} / 312^{\prime} \times 2^{\prime}$ field by Kodaira et al. (1998b) as possible candidates for the superwind-phase AGB stars, we find $n_{j} \sim 7$ in the $\mathrm{f} / 312^{\prime} \times 2^{\prime}$ field and $\log t_{j} \sim 4.9$. When we take the uncertainty of the mixed population noted above into account, this may be in broad agreement with the estimate $\log t_{j} \sim 5$ by Tanabe et al.(1997) for similar objects in the intermediate-age clusters in the LMC, to support their inference about the high mass-loss rate of these objects.

The present star formation rate in the observed $7^{\prime} \times 7^{\prime}$ domain over the last $\sim 10$ Myr is estimated at $\sim 1.8 \times 10^{4} m_{\odot} \mathrm{Myr}^{-1}$ per deprojected disk area of $1 \mathrm{kpc}^{2}$ for an inclination of $i=12 .^{\circ} 5$. This estimate is derived from the number of the young stars of $-4.5 \lesssim M_{V} \lesssim-3.5$ corresponding to $\sim$ B0 V stars of $\sim 15-19 m_{\odot}, n=9 \operatorname{arcmin}^{-2} \mathrm{mag}^{-1}$, by adopting the Salpeter initial mass function for the mass range of $0.1-120 m_{\odot}$. As was pointed out in the last section, the ratio of the young population to the old population varies among subdomains, suggesting local variation in the star formation rate. The local 
intensification factors of the star formation rate over the last $\sim 10$ Myr are derived from the number of stars in the range $-4.5 \lesssim M_{V} \lesssim-3.5$ and are estimated at $\sim 4, \sim 2, \sim 1$, and $\sim 0.5$ times the above mean value $\left(\sim 1.8 \times 10^{4} m_{\odot} \mathrm{Myr}^{-1} \mathrm{kpc}^{-2}\right)$ in the subdomains 17, 15, 19, and 4, respectively (see Fig. 7).

Additionally, we obtain an estimate on the average star formation rate $\sim 7.2 \cdot 10^{3} \mathrm{~m}_{\odot}$ $\mathrm{Myr}^{-1}$ per deprojected area of $1 \mathrm{kpc}^{2}$ from the observed star number $n=70 \mathrm{arcmin}^{-2}$ $\mathrm{mag}^{-1}$ of the bright AGB stars of $-4.8 \lesssim M_{b o l} \lesssim-3.8$ in the $3^{\prime} \times 3^{\prime}$ field. Thereby we assume that these stars have ages of $\sim 0.2-2.5$ Gyr (corresponding to the mass of $\sim 4.0-1.5 m_{\odot}$ ) and the standard lifetime of $1.3 \mathrm{Myr} \mathrm{mag}^{-1}$. The bright AGB stars of $-4.8 \lesssim M_{b o l} \lesssim-3.8$ may have drifted from their birth volume because of the proper motion and the resulting epicyclic motion, and their distribution is correspondingly smeared out relative to the young stars with $-4.5 \lesssim M_{V} \lesssim-3.5$. The star formation rate derived from the bright $\mathrm{AGB}$ stars may reflect the average rate in the disk at $R \simeq 7 \mathrm{kpc}$ rather than the specific domain related to A24. These star formation rates slightly increase when the larger distance modulus $(m-M=24.47)$ or/and a stronger interstellar extinction would be assumed. The above star formation rates for the M31 field around A24 may be compared with the present star formation rates in the Galaxy, $(3.5-5.0) \times 10^{3} m_{\odot} \mathrm{Myr}^{-1} \mathrm{kpc}^{-2}$ for the $1 \mathrm{kpc}$ vicinity of the Sun, and $8 \times 10^{3} m_{\odot} \mathrm{Myr}^{-1} \mathrm{kpc}^{-2}$ for the disk in average at $R \simeq 7$ kpc (see Rana 1991). This comparison suggests a similar level of the present star formation activity between the average Galactic disk and the M31 field around A24 on a spiral arm, and consequently may indicate a slightly low star formation activity in the M31 disk in average relative to the Galactic disk around $R=7 \mathrm{kpc}$. The relatively low present star formation activity in M31 was pointed out by previous investigators (see Tomita, Tomita, \& Saitō 1996 and references therein). The present star formation rate of a whole galaxy was estimated at $0.8-13 m_{\odot} \mathrm{yr}^{-1}$ for the Galaxy (Rana 1991 and references therein) and at $0.2-0.5 m_{\odot} \mathrm{yr}^{-1}$ for M31 (Walterbos 1988), based upon various total emissions such as 
$\mathrm{H} \alpha$ and the far-infrared.

A kind of anticorrelation between the young population and the intermediate-age population, which was found in the last section, may suggest that the star formation in individual disk subdomains of a size $\sim 100-200$ pc was not continuous but might have been intermittent, like a "Christmas tree" model. An inspection of CMDs for the different groups in Figures 10a and 10b suggests that the deficient AGBs are mainly those of $t \lesssim 0.5$ Gyr. The history of the local star formation may be smeared out over a timescale longer than this. The rotation period of the disk domain of $R=4-10 \mathrm{kpc}$ is $\sim 0.2 \mathrm{Gyr}$, and the period of the spiral-pattern passage is almost the same in the case of a two-arm spiral (Braun 1991). Some subdomains of the stellar complex A24 have just experienced star formation in the present spiral passage but did not in the last few passages. Some other subdomains actively formed stars in the last passages one or two times $\sim 0.2$ Gyr ago but not in the present passage. Disk domains of $\sim 100-200$ pc size seem to be filled up with cool gas sufficient to produce stars again on a timescale of a few times the spiral passage period.

The actual dust and gas clouds in the studied field have complex structures. The deep $V$-band image (Fig. 1a) reveals fine complex textures of dark clouds surrounding the clusters C202 and C203 in the superbubble, with various scales of $1^{\prime}-0 .^{\prime} 1(200-20$ pc) down to $1^{\prime \prime}(\sim 3 \mathrm{pc})$ order of spotlike features that might be cores or knots of large clouds. Individual early-type stars show a wide range of interstellar extinction of $A_{V} \simeq 0-3$, again indicating highly local inhomogeneities. The result of the analysis of the local luminosity function in the $V$ band also supports the inhomogeneous nature of the absorbing clouds. The real three-dimensional structure of this stellar complex with absorbing clouds is difficult to model uniquely because of the awkward projection angle $i=12 .^{\circ} 5$ of M31. The fine structures are not particularly elongated along the major axis. Some fibrous features are 
even oriented along the minor axis, or perpendicular to the direction of the spiral arm. Accordingly, we suspect that the dark cloud complex surrounding the superbubble around C202 and C203 is not perfectly flat in the central plane but is equally extended above the plane, as was pointed out for dark lanes in some edge-on spiral galaxies by Sofue (1987) and Sofue, Wakamatsu, \& Malin (1994).

The spiral dark lane appears to be broken on both sides of D221, but the ridge rich in young stars runs through it along the spiral arm (Figs. 1 and 6). Although the detailed three-dimensional structures of the clouds are not clear, Figure 6 (see also Fig. 1b) gives an impression for the global trend around the $7 \mathrm{kpc}$ spiral-arm structure that the distribution of the young stellar population shows a wide ridge which seems to be slightly shifted outward relative to that of the absorbing clouds, conforming to the view about the sequential distribution of the interstellar matter and the newly born stars for the case of the trailing spiral; this was suggested by Loinard et al. (1996) in the study of the distribution of $\mathrm{CO}$ and $\mathrm{HI}$ clouds relative to that of H II regions in the M31 spiral arms.

In conclusion, we believe that this work has demonstrated the possibility of detailed population analysis of M31, and we would like to underline the importance of extensive deep photometry in the visual as well as in the infrared region.

The authors wish to acknowledge the collaborative assistance of the staff members of the University of Hawaii Institute for Astrophysics during the observation at Mauna Kea, and the generous cooperation in the IRAS data processing by the Infrared Processing and Analysis Center operated by CIT/JPL under the NASA contract. We are grateful to A. Kučinskas for providing us with the data for deriving the bolometric correction prior to publication. The computational work was done using the facilities of the Astronomical Data Analysis Computing Center of NAOJ. This work was partially supported by Grant in Aid for Science Research 07640360. 


\section{APPENDIX}

\section{A. Bolometric Correction}

In order to transform $M_{K}$ to $M_{b o l}$, we used the bolometric correction $\left(B C_{K}\right)$ as a seventh-order polynomial function of $(J-K)_{0}: B C_{K}=\Sigma a_{i}(J-K)_{0}^{i}$, with the following coefficients: $a_{0}=0.24, a_{1}=3.89, a_{2}=-1.795, a_{3}=0.586, a_{4}=-0.241, a_{5}=0.0661$, $a_{6}=-0.00859, a_{7}=0.00041$, for $-0.1 \leq(J-K)_{0} \leq 3.5$. The polynomial was derived by fitting to the empirical relation for giants in globular clusters in the Milky Way $\left[-0.1 \leq(J-K)_{0} \leq 1.4\right]$ by Montegriffo et al. (1998), $B C_{K}$ values of individual AGB stars

in the South Galactic Cap $\left[1.1 \leq(J-K)_{0} \leq 2.5\right]$ from Whitelock et al. $(1994,1995)$, and $B C_{K}$ values of the $\mathrm{OH} / \mathrm{IR}$ stars $\left[1.1 \leq(J-K)_{0} \leq 3.5\right]$ from Kučinskas (1998), which were corrected by $\Delta B C_{K}=+0.5$ to match others in the range $1<(J-K)_{0}<2$; see Figure 11 . In the latter two sources, the interstellar reddening $E(J-K)$ was regarded as negligible compared with the intrinsic dispersion. Although the sources were heterogeneous as to the age and the metallicity, we believe that this empirical correction incorporated the essential effects of the extinction and the thermal emission by the hot dust shells, and that this transformation is superior to those used in previous works which assumed almost constant $B C_{K} \simeq 3.25$ for $(J-K)_{0}>1.5$.

\section{B. Correction for the Dust-Shell}

The dust-shell structure and the radiative transfer through the shell were calculated with the program DUSTY by Ivezić, Nenkova, \& Elitzur (1997). The density profile of the stationary shell was calculated for the varying wind velocity which was accelerated by the radiation pressure on dust, and the dust temperature at the inner boundary of the shell 
was taken to be $800 \mathrm{~K}$. The dust opacity was calculated for the silicate parameters given in Draine \& Lee (1984), in contrast to the special mixtures in Bressan et al. (1998), and for the size distribution as adopted by Mathis, Rumpl, \& Nordsieck (1977). Models were calculated for the optical thickness of the shell $\tau_{V}=1,3,10,30$, and 100, and for the central blackbody source of temperature $T=2000,2500,3000$, and $3500 \mathrm{~K}$. By comparing the original blackbody SED with the SED of the corresponding shell model, we evaluated the corrections in the near-infrared photometric magnitudes. These corrections were applied to the theoretical isochrones by the Padova group (Bertelli et al. 1994) according to the following conditions. The dust-shell was assumed to become effective at $M_{b o l}=-3.8$ with $\tau_{V}=1$. If this point is too hot for relatively young populations (age $100-200 \mathrm{Myr}$ ), the first shell-effective point was assumed to be the point on the original isochrone in the $M_{b o l}$ versus $(H-K)_{0}$ diagram (Fig. $5 \mathrm{c}$ ), where the isochrone deviates from the vertical path. The thickness of the dust-shell was assumed to be $\tau_{V}=30$ at the tip of the AGB, and the corrections were linearly interpolated between the first point $\left(\tau_{V}=1\right)$ and the final one $\left(\tau_{V}=30\right)$ proportional to $M_{b o l}$. The case of $\tau_{V} \leq 10$ for the tip of AGB turned out to be insufficient, while the case of $\tau_{V}=100$ produces too red AGB isochrones to match the observation. The lower limit of $M_{b o l}=-3.8$ and the linear $M_{b o l}-\tau_{V}$ relation were deduced from the data of O-rich and C-rich stars (Kučinskas 1998). 


\section{REFERENCES}

Battaner, E., Beckman, J. E., Mediavilla, E., Prieto, M., Sanchez Magro, C., Muñoz Tuñon, C., \& Sanchez Saavedra, M. L. 1986, A\&A, 161, 70

Bertelli, G., Bressan, A., Chiosi, C., Fagotto, F., \& Nasi, E. 1994, A\&AS, 106, 275 (Padova 94)

Bessell, M. S., \& Brett, J. M. 1988, PASP, 100, 1134

Braun, R. 1991, ApJ, 372, 54

Bressan, A., Granato, G. L., \& Silva, L. 1998, A\&A, 332, 135

Brinks, E., \& Shane, W. W. 1984, A\&AS, 55, 179

Burstein, D., \& Heiles, C. 1984, ApJS, 54, 33

Devereux, N. A., Price, R., Wells, L. A., \& Duric, N. 1994, AJ, 108, 1667

Draine, B. T., \& Lee, H. M. 1984, ApJ, 285, 89

Efremov, Yu. N., Ivanov, G. R., \& Nikolov, N. S. 1987, Ap\&SS, 135, 119

Haiman, Z., et al. 1994, A\&A, 290, 371

Hiromoto, N., Maihara, T., Oda, N., \& Okuda, H. 1983, PASJ, 35, 413

Hodge, P. W. 1981, Atlas of the Andromeda Galaxy (Seattle and London: Univ. Washington Press)

Hodge, P. W., \& Lee, M. G. 1988, ApJ, 329, 651

Holland, S. 1998, AJ, 115, 1916

Humphreys, R. M. 1979, ApJ, 234, 854 
Huterer, D., Sasselov, D. D., \& Schechter, P. L. 1995, AJ, 110, 2705

Iben, I., \& Renzini, A. 1983, ARA\&A, 21, 271

Ivezić, Ž., Nenkova, M., \& Elitzur, M. 1997, User Manual for DUSTY (Univ. Kentucky)

Kent, S. M. 1989, AJ, 97, 1614

Kerschbaum, F., Lazaro, C., \& Habison, P. 1996, A\&AS, 118, 397

Kodaira, K., Miyazaki, S., Vansevičius, V., Tamura, M., Tokunaga, A., \& Kobayashi, N. 1998a, ApJS, 118, 177 (Paper I)

Kodaira, K., Tamura, M., Vansevičius, V., \& Miyazaki, S. 1998b, ApJ, 500, L133

Kučinskas, A. 1998, Ph.D. Thesis, Institute of Theoretical Physics and Astronomy (Lithuania)

Loinard, L., Dame, T. M., Koper, E., Lequeux, J., Thaddeus, P., \& Young, J. S. 1996, ApJ, 469, L101

Magnier, E. A., et al. 1993, A\&A, 278, 36

Magnier, E. A., et al. 1995, A\&AS, 114, 215

Magnier, E. A., Hodge, P., Battinelli, P., Lewin, W. H. G., \& van Paradijs, J. 1997, MNRAS, 292, 490

Magnier, E. A., Lewin, W. H. G., van Paradijs, J., Hasinger, G., Jain, A., Pietsch, W., \& Trümper, J. 1992, A\&AS, 96, 379

Martinez Roger, C., Phillips, J. P., \& Sanchez Magro, C. 1986, A\&A, 161, 237

Mathis, J. S., \& Cardelli, J. A. 1990, BAAS, 22, 861 
Mathis, J. S., Rumpl, W., \& Nordsieck, K. H. 1977, ApJ, 217, 425

Montegriffo, P., Ferraro, F. R., Origlia, L., \& Fusi Pecci, F. 1998, MNRAS, 297, 872

Pellet, A., Astier, N., Viale, A., Courtes, G., Maucherat, A., Monnet, G., \& Simien, F. 1978, A\&AS, 31, 439

Pritchet, C. J., \& van den Bergh, S. 1987, ApJ, 316, 517

Rana, N. 1991, ARA\&A, 29, 129

Renzini, A., \& Buzzoni, A. 1986, in Spectral Evolution of Galaxies, ed. C. Chiosi \& A. Renzini (Dordrecht: Reidel), 195

Rich, R. M., Mould, J. R., \& Graham, J. R. 1993, AJ, 106, 2252

Sandage, A., \& Tammann, G. A. 1968, AJ, 151, 531

Sofue, Y. 1987, PASJ, 39, 547

Sofue, Y., Wakamatsu, K., \& Malin, D. F. 1994, AJ, 108, 2102

Stanek, K. Z., \& Garnavich, P. M. 1998, ApJ, 503, L131

Tanabe, T., et al. 1997, Nature, 385, 509

Tomita, A., Tomita, Y., \& Saitō, M. 1996, PASJ, 48, 285

Unwin, S. C. 1980, MNRAS, 190, 551

van den Bergh, S. 1964, ApJS, 9, 65

Walterbos, R. A. M. 1988, in Galactic and Extragalactic Star Formation, ed. R. E. Pudritz \& M. Fich (Dordrecht: Kluwer), 361

Welch, D. L., McAlary, C. W., McLaren, R. A., \& Madore, B. F. 1986, ApJ, 305, 583 
Whitelock, P., Menzies, J., Feast, M., Catchpole, R., Marang, F., \& Carter, B. 1995, MNRAS, 276, 219

Whitelock, P., Menzies, J., Feast, M., Marang, F., Carter, B., Roberts, G., Catchpole, R., \& Chapman, J. 1994, MNRAS, 267, 711

Xu, C., \& Helou, G. 1996a, ApJ, 456, 152

Xu, C., \& Helou, G. 1996b, ApJ, 456, 163 


\section{FIGURE CAPTIONS}

Fig. 1.- (a) $V$-band image showing the whole of the $7^{\prime} \times 7^{\prime}$ area studied around A24. (b) Relative locations of the $7^{\prime} \times 7^{\prime} V$ field, the $3^{\prime} \times 3^{\prime} J H$ field, and the $2^{\prime} \times 2^{\prime} J H K$ field. The Hodge Atlas is underlaid. The frame of the $7^{\prime} \times 7^{\prime}$ field is slightly inclined relative to others which are oriented with north at the top and east to the left.

Fig. 2. $-V$ luminosity function for the $7^{\prime} \times 7^{\prime}$ field. $A_{V}=1.0$ is assumed.

Fig. 3.- Bolometric luminosity function for the $2^{\prime} \times 2^{\prime} J H K$ field.

Fig. 4. - Two-color diagram for bright stars $(K<18.0)$ in the $2^{\prime} \times 2^{\prime} J H K$ field. The isochrones are those modified using dust-shell models; see text. The ages of the isochrones are 10, 20, 50, 100, 200, and 500 Myr (AGB tracks with curved branching-off), and 5 and 10 Gyr (AGB tracks with straight branching-off), but they are almost degenerated. The oldest two cases are for the helium and metal fractions $Y=0.25$ and $Z=0.008$, and the others are for $Y=0.28$ and $Z=0.02$. Only the RGB tracks for the oldest two cases extend to the reddest part of $(H-K)_{0}>0.5$. A reddening vector for $A_{V}=3$ is indicated according to the Galactic extinction law. Original isochrones of Padova 94 are strongly degenerated, and that for the age of $100 \mathrm{Myr}$ only is shown in the inset with IRAS AGB stars of Whitelock et al. $(1994,1995)$.

Fig. 5.- Color-magnitude diagrams for stars in the $2^{\prime} \times 2^{\prime} J H K$ field. The isochrones are those modified using dust-shell models; see text. The ages of the isochrones are 10, 20, 50, 100, 200, and $500 \mathrm{Myr}$ and 1, 2, 5, and 10 Gyr (from top to bottom). The oldest two cases are for the helium and metal fractions $Y=0.25$ and $Z=0.008$, and the others are for $Y=0.28$ and $Z=0.02$. A dereddening vector for $A_{V}=5$ is indicated in (a), according to the Galactic extinction law. Original isochrones of Padova 94 are shown in the insets for the same set of ages and metallicities as those in the main frames. 
Fig. 6. - Subdomains in the $7^{\prime} \times 7^{\prime} V$ field for which luminosity functions were differentially studied. The analyzed subdomains are numbered from 1 to 33 and are indicated by a different number of asterisks and darkness according to the extinction index $\Delta V$ and the population ratio index $r_{V}$ : no asterisk means $\Delta V<0.1$; a single asterisk, $0.1 \leq \Delta V<0.25$; two asterisks, $0.25 \leq \Delta V \leq 0.5$. Decreasing degrees of darkness are for $r_{V}>1.0,0.5 \leq r_{V} \leq 1.0,0.25<r_{V} \leq 0.5$, and $r_{V} \leq 0.25$, respectively. The clear mesh fields are not combined into the subdomains. See text for the definitions of $\Delta V$ and $r_{V}$.

Fig. 7.- Typical examples of the magnitude distributions for subdomains having different population ratio indices $r_{V}$ : subdomains 4 (circles), 19 (downward-pointing triangles), 15 (crosses), and 17 (upward-pointing triangles) having $r_{V}=0.10,0.31,0.58$, and 1.1, respectively. The curves for subdomains 4, 19, and 15 are vertically shifted relative to that of subdomain 17 by multiplying by factors of $0.80,1.09$, and 1.03 , respectively. The straight lines show the gradients 0.5 and 2.24. See text for the definition of $r_{V}$.

Fig. 8.- Typical magnitude distributions for subdomains having different extinction indices $\Delta V$. See text for the definition of $\Delta V$. (a) Subdomain 6 (triangles) and the reference subdomain 15 (circles). (b) Subdomain 26 (triangles) and the reference subdomain 28 (circles). The curves for subdomains 6 and 26 are horizontally shifted by $\Delta V=-0.4$.

Fig. 9.- Subdomains in the $3^{\prime} \times 3^{\prime} H$ field for which luminosity functions were differentially studied. The analyzed subdomains are numbered from 1 to 15 and are shaded according to the population ratio index $r_{H}$. Decreasing degrees of darkness are for $r_{H}>1$, $0.7<r_{H} \leq 1$, and $0.5<r_{H} \leq 0.7$, respectively. The clear mesh fields are not combined into subdomains. See text for the definition of $r_{H}$.

Fig. 10.- Typical magnitude distributions for subdomains having different population ratio indices $r_{H}$. (a) $J$ and (b) H. "High" subdomains $r_{H} \sim 1.0$ (Nos. 2, 4, 5, and 15; upward-pointing triangles), "medium" subdomains $r_{H} \sim 0.7$ (Nos. 3, 10, 12, and 
13; circles), and "low" subdomains $r_{H} \sim 0.5$ (Nos. 7, 9, 11, and 14; downward-pointing triangles). See text for the definition of $r_{H}$.

Fig. 11.- Polynomial fitting of $B C_{K}$ as function of $(J-K)_{0}$ to the empirical relation by Montegriffo et al. (1998) (triangles), and the data of AGB stars in Whitelock et al. (1994, 1995) (circles) and OH/IR stars in Kučinskas (1998) (dots). The Kučinskas data were shifted by $0.5 \mathrm{mag}$ upward to match others in the range $1<(J-K)_{0}<2$. In the latter two sources, the reddening correction was assumed to be negligible compared to the intrinsic dispersion. 
This figure "FIG_1A.JPG" is available in "JPG" format from: http://arxiv.org/ps/astro-ph/9903029v1 
This figure "FIG_1B.JPG" is available in "JPG" format from: http://arxiv.org/ps/astro-ph/9903029v1 


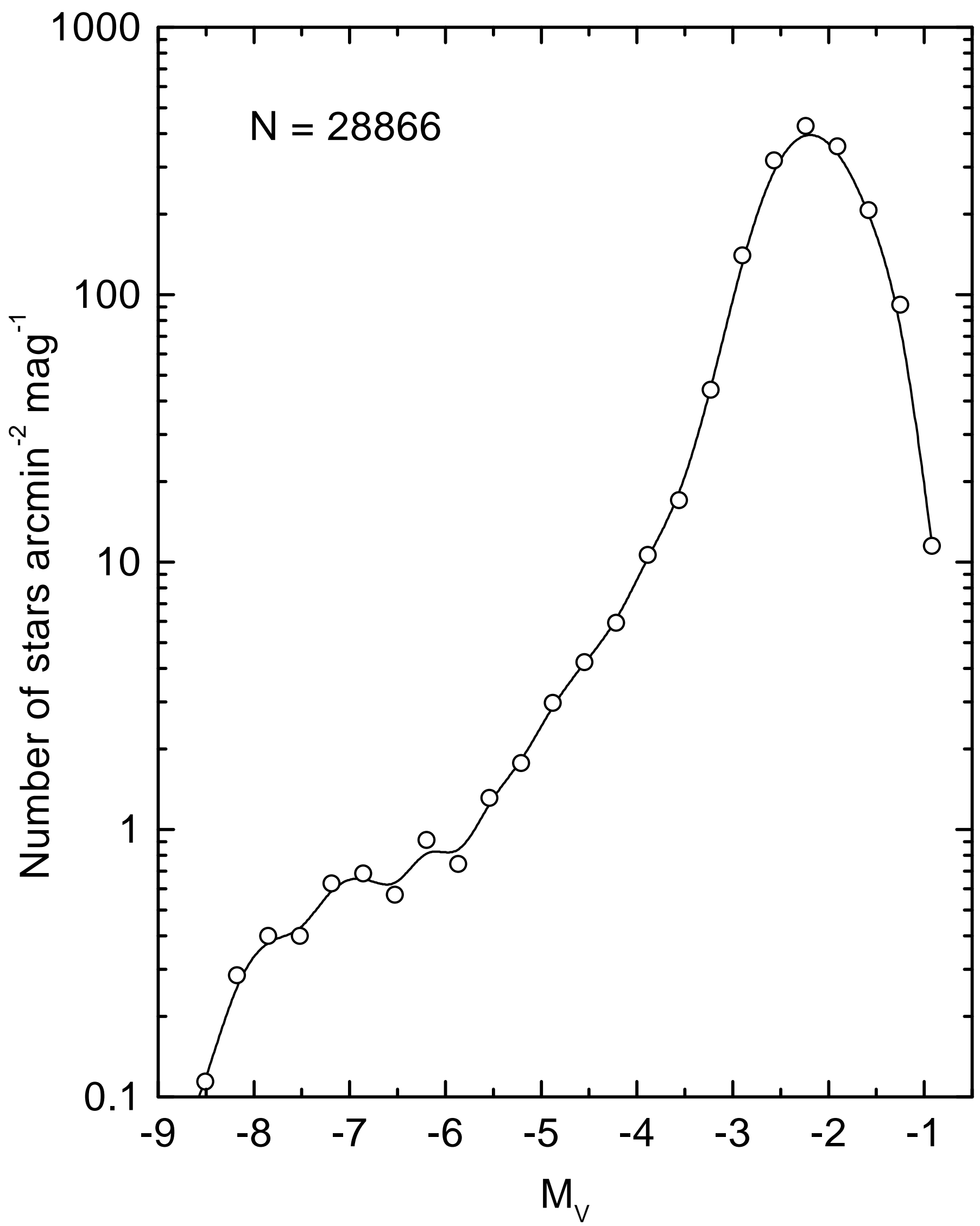




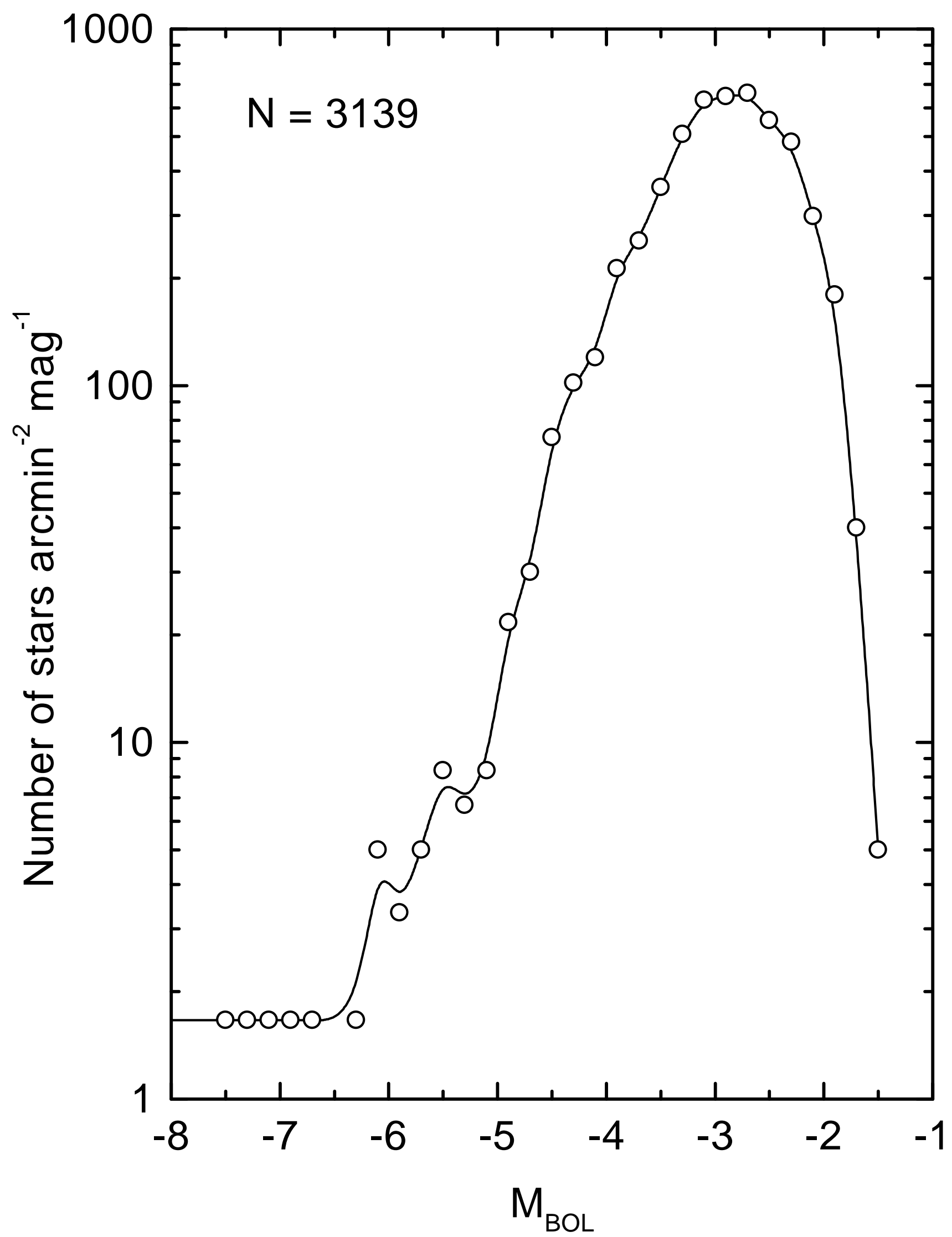




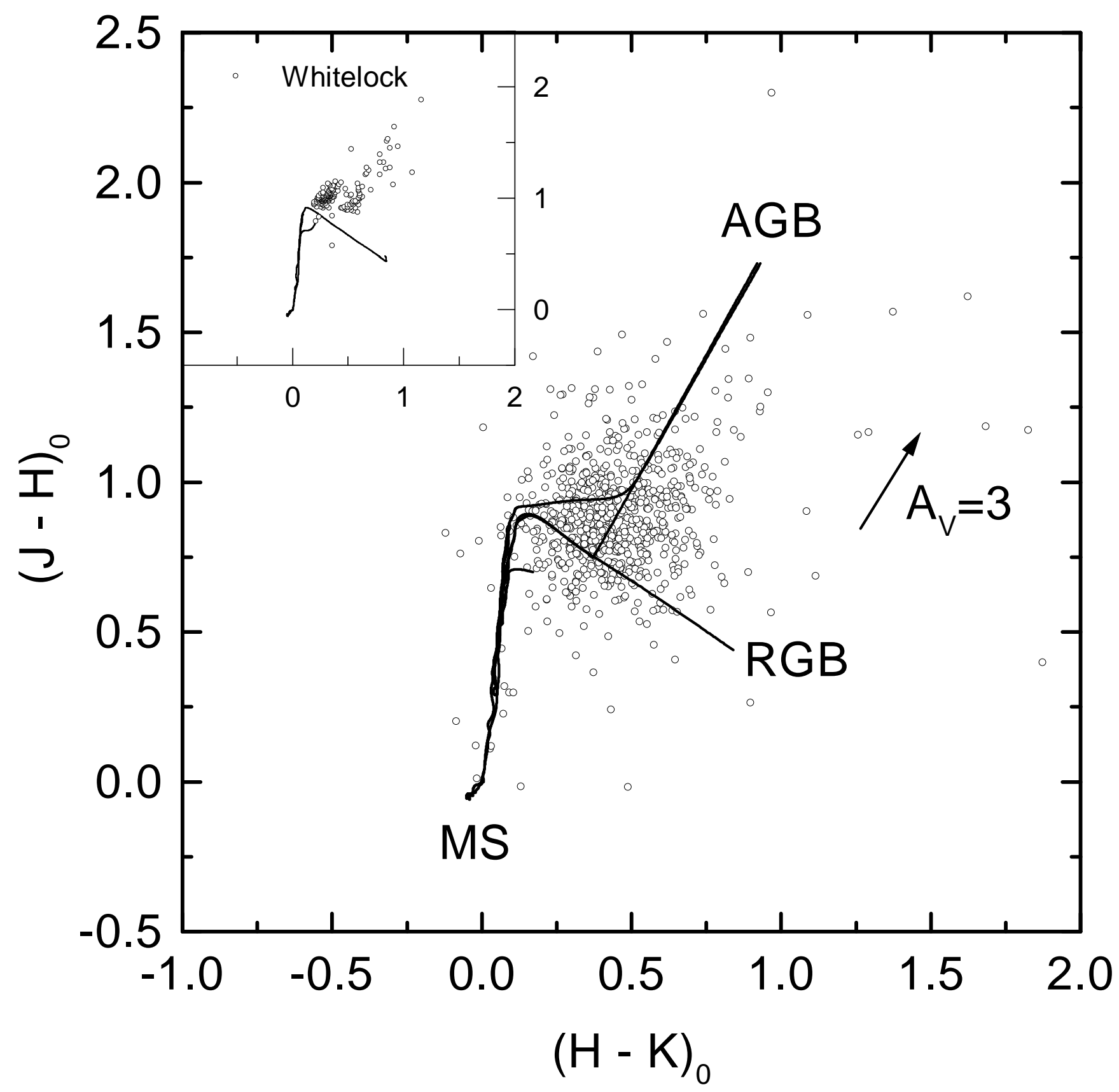




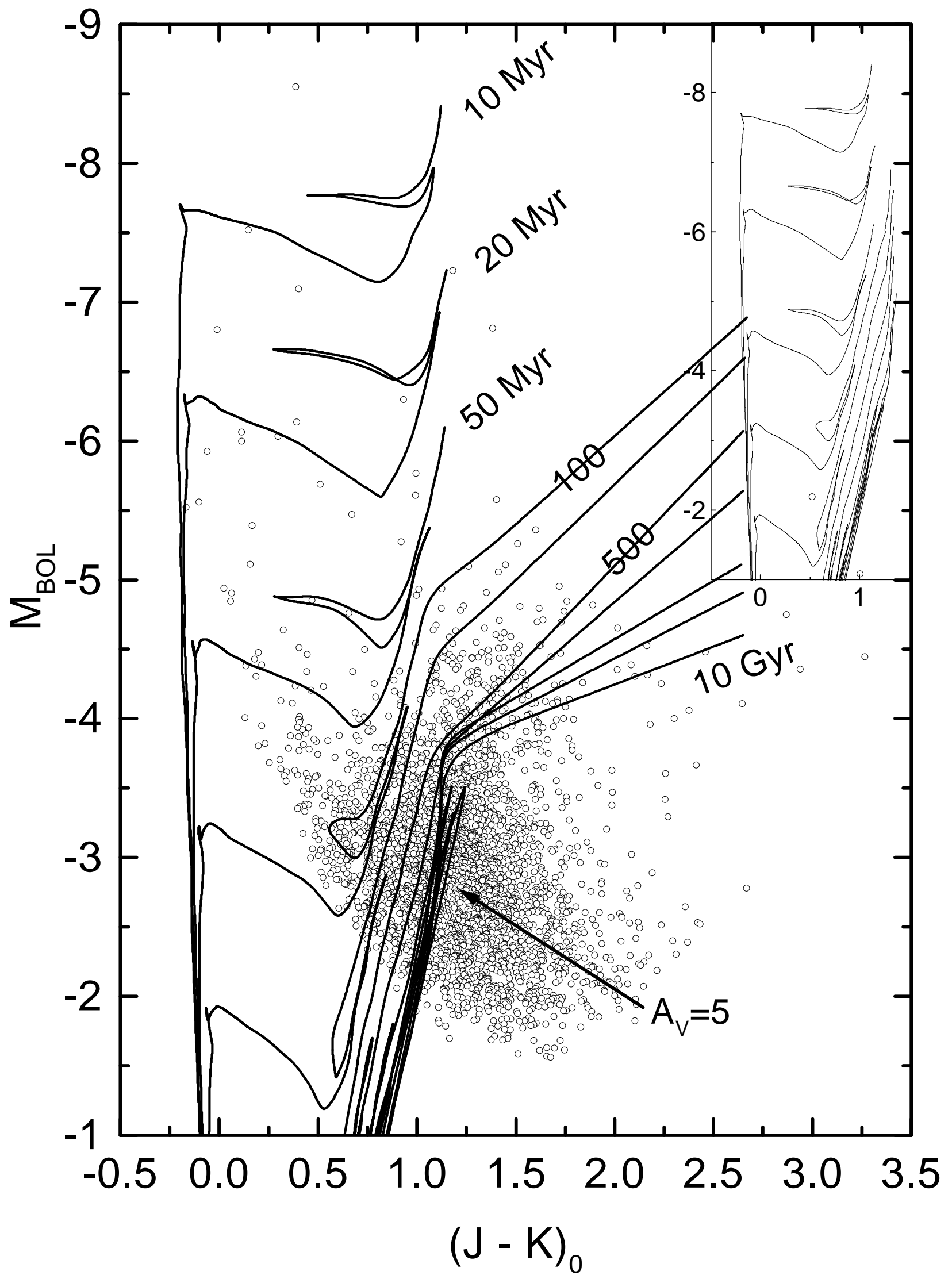




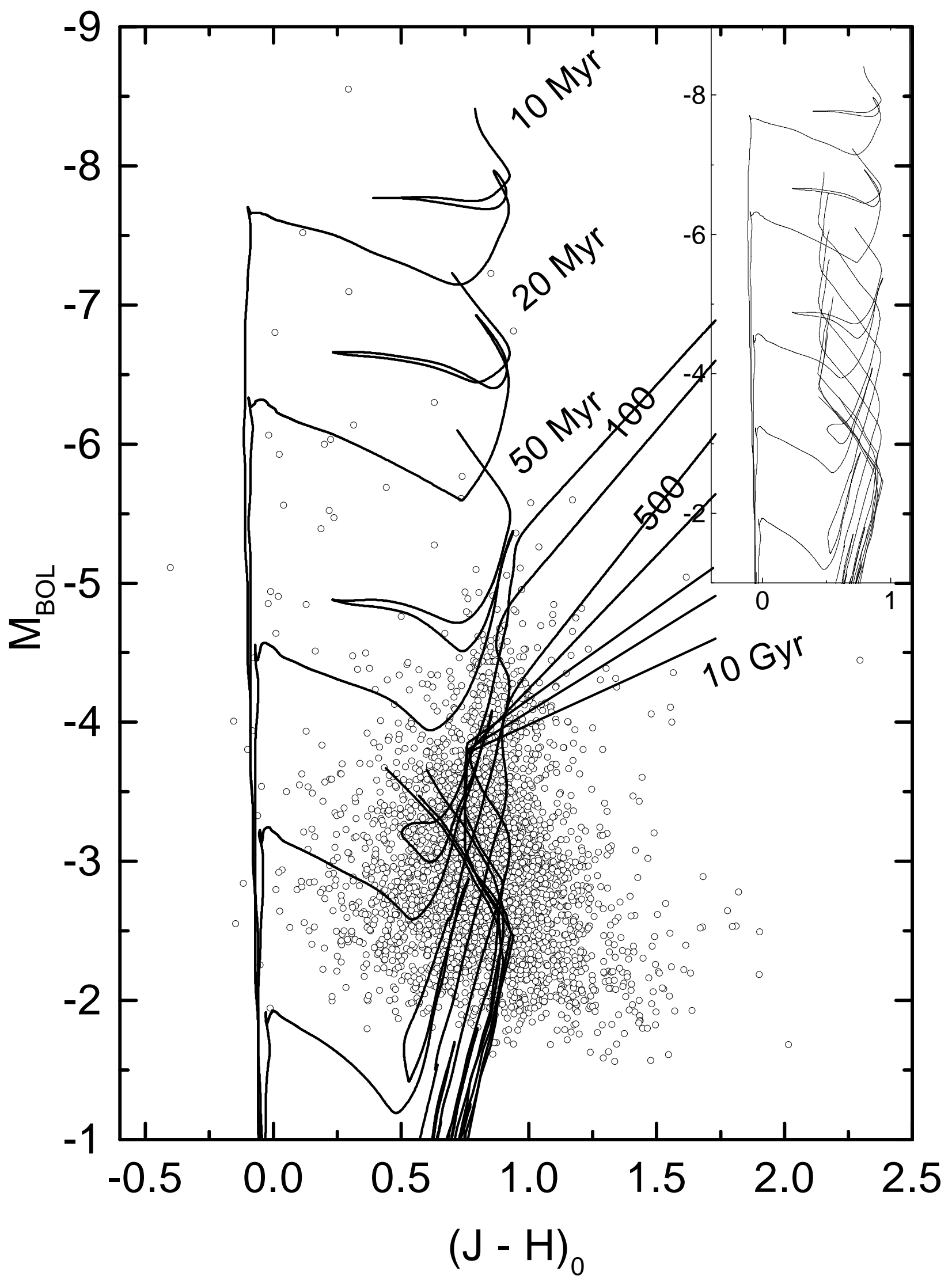




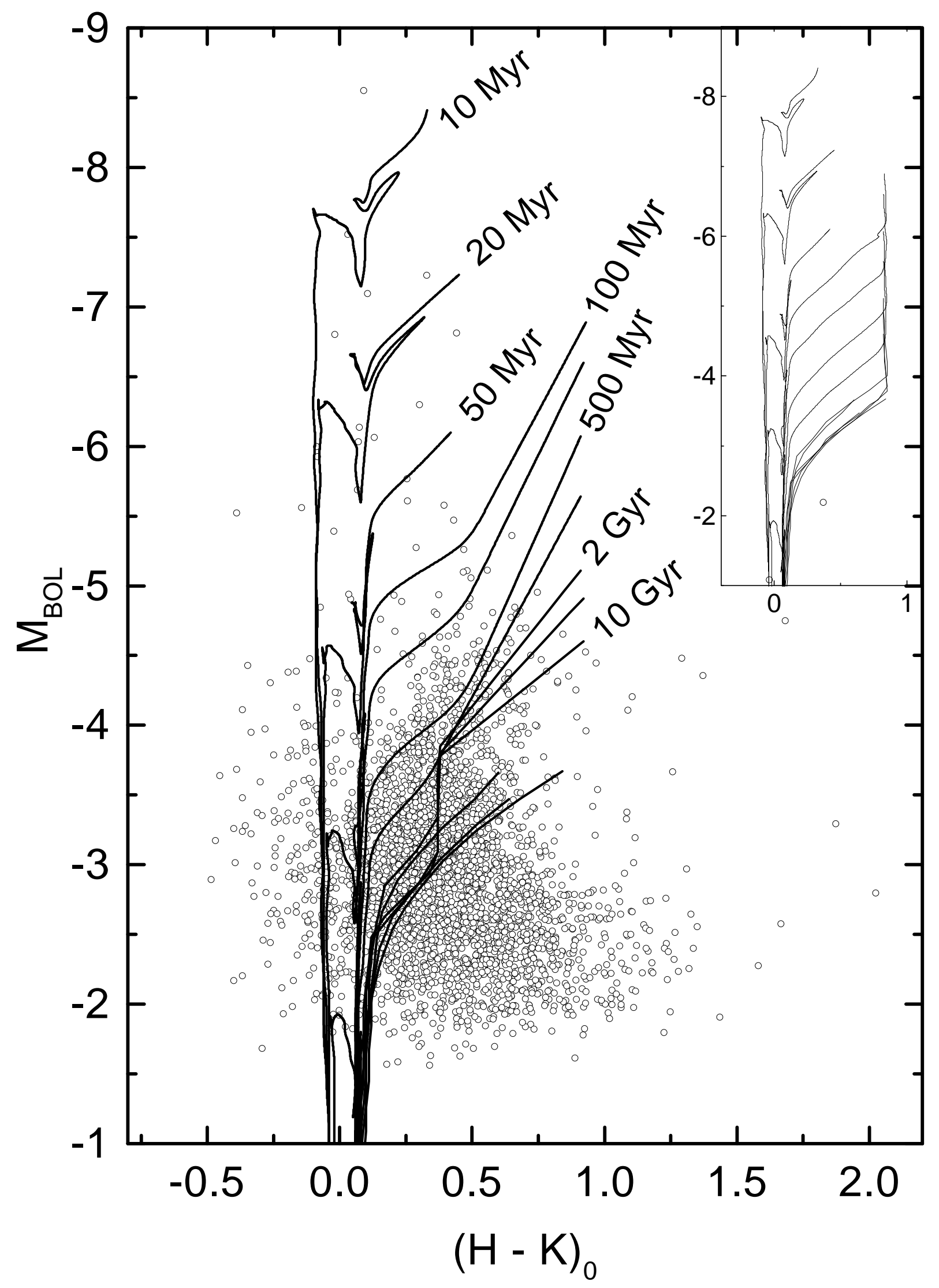


This figure "FIG6.JPG" is available in "JPG" format from: http://arxiv.org/ps/astro-ph/9903029v1 


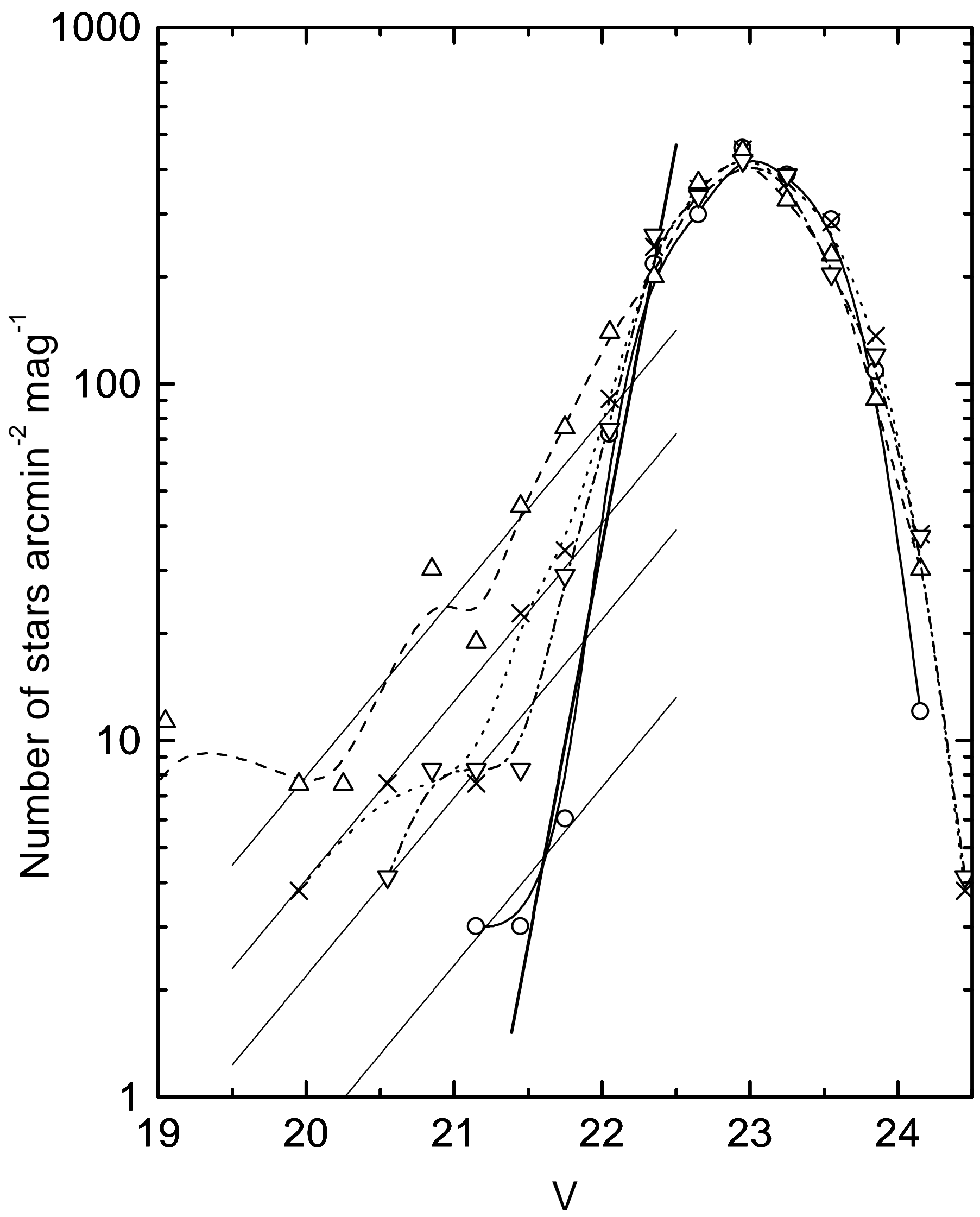




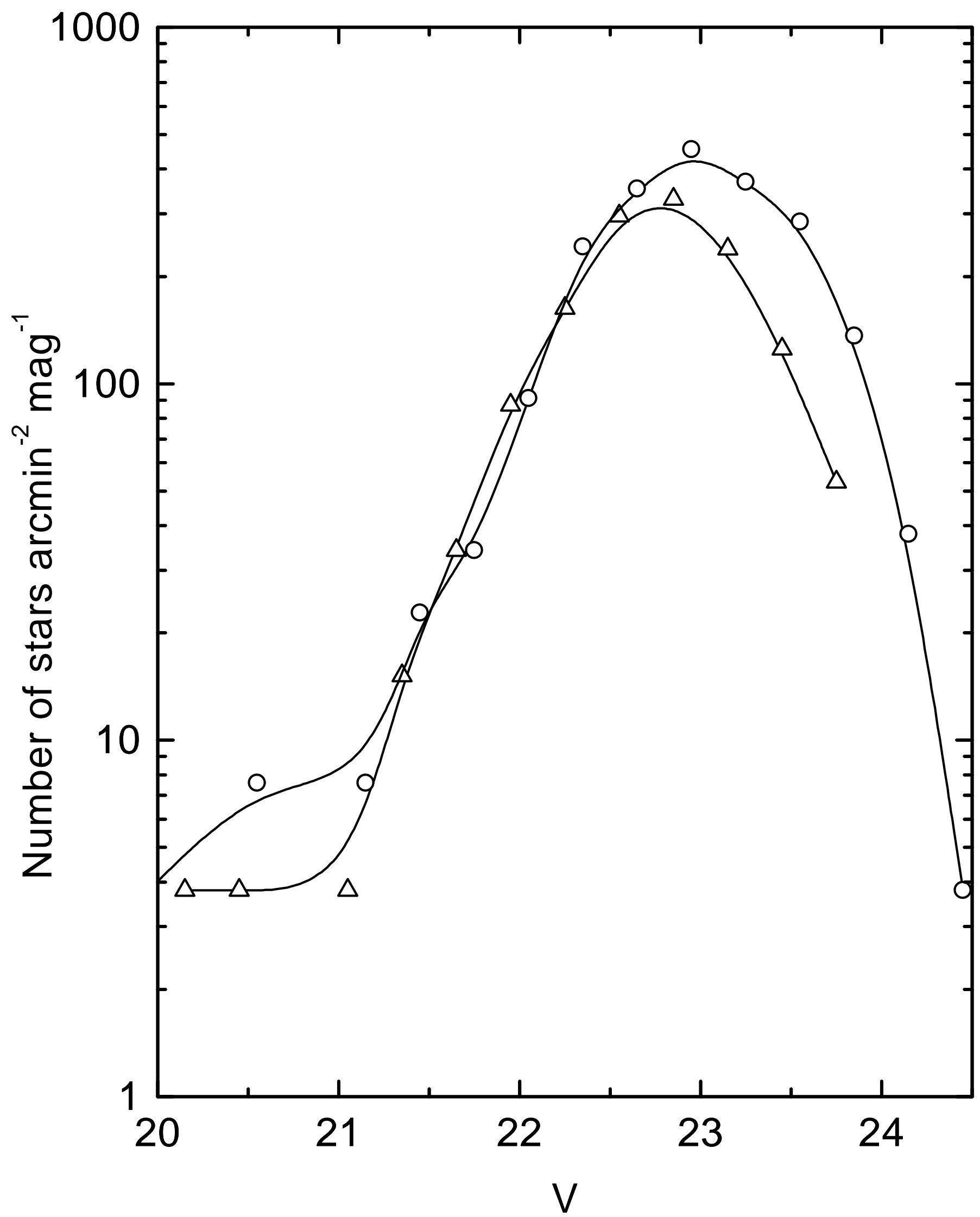




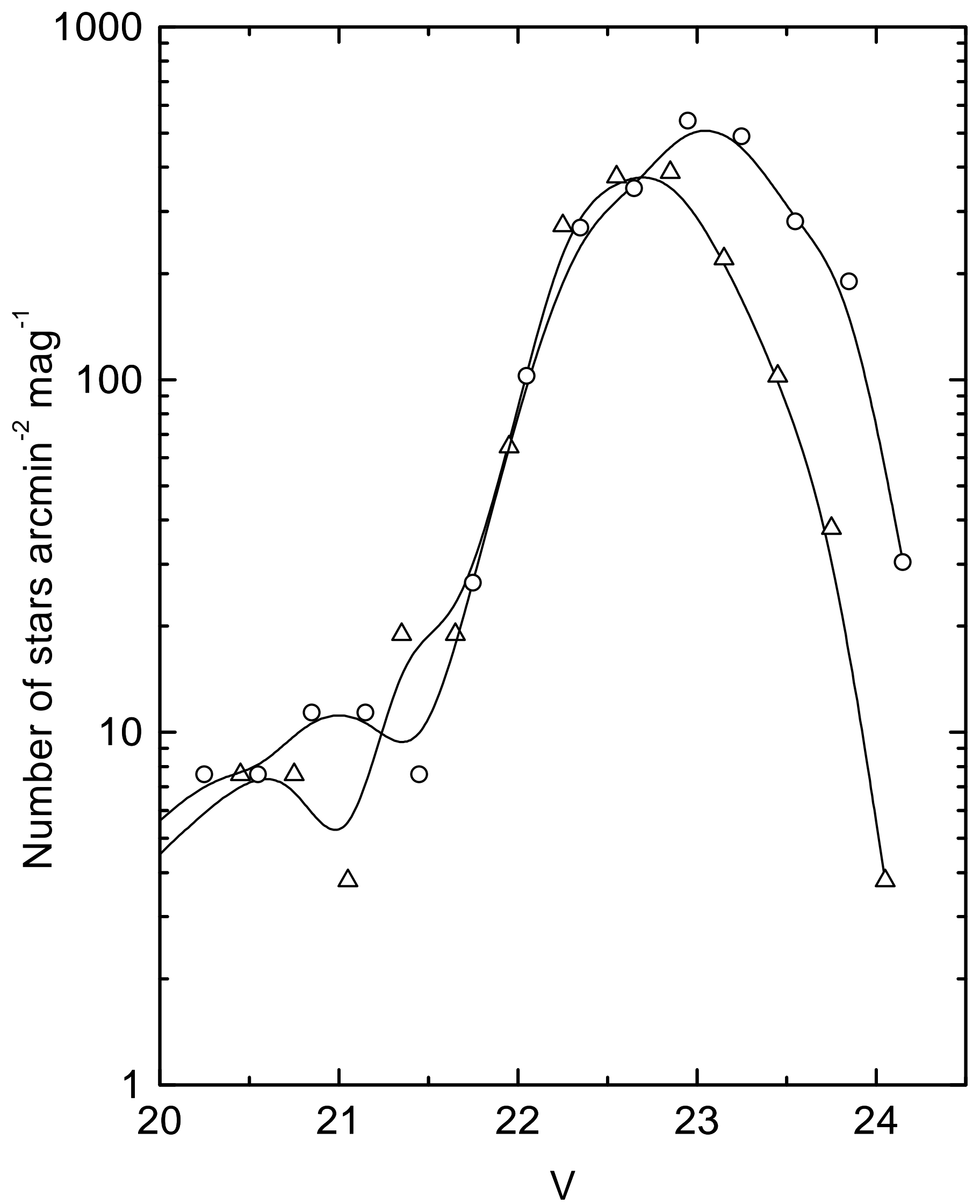


This figure "FIG9.JPG" is available in "JPG" format from: http://arxiv.org/ps/astro-ph/9903029v1 


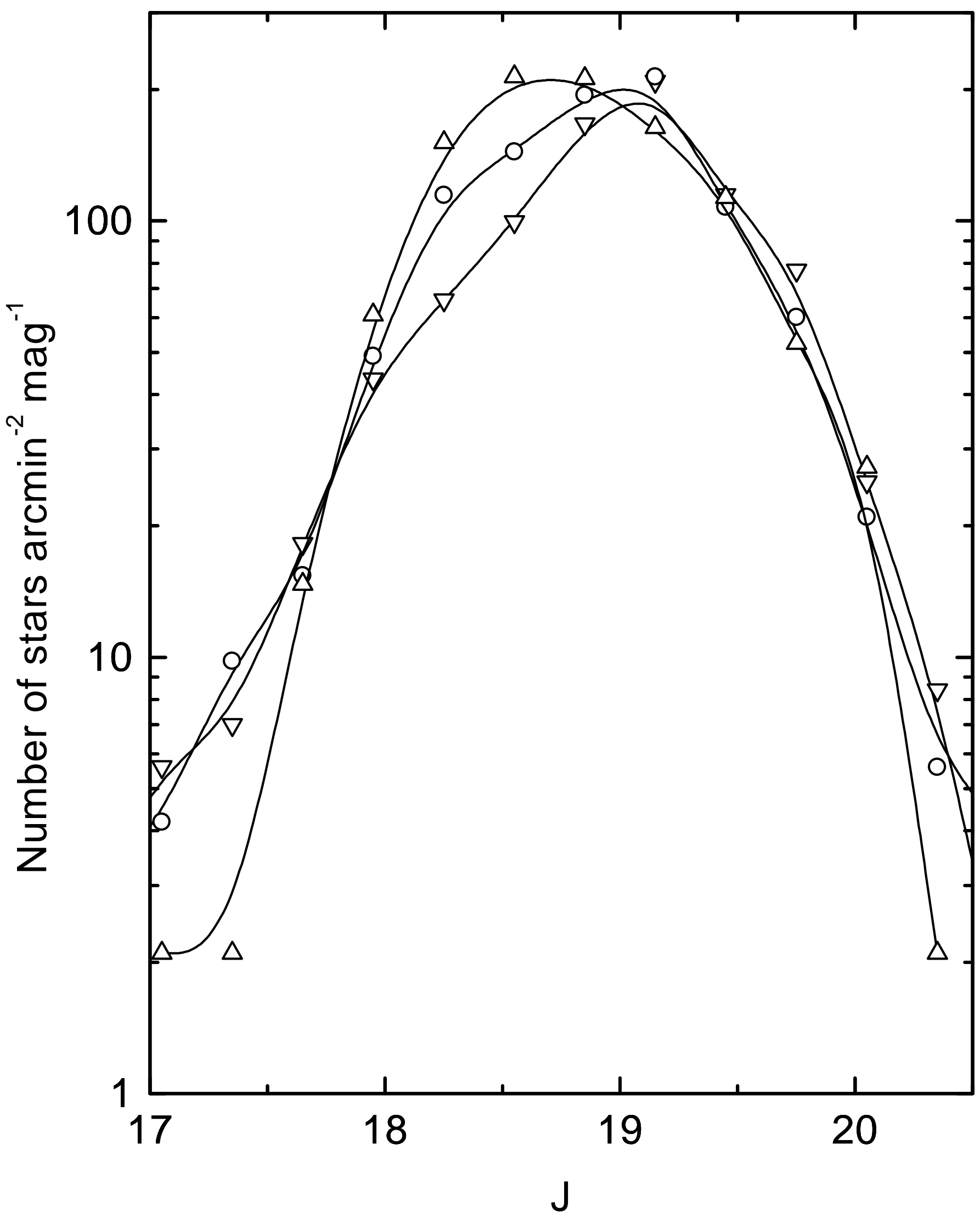




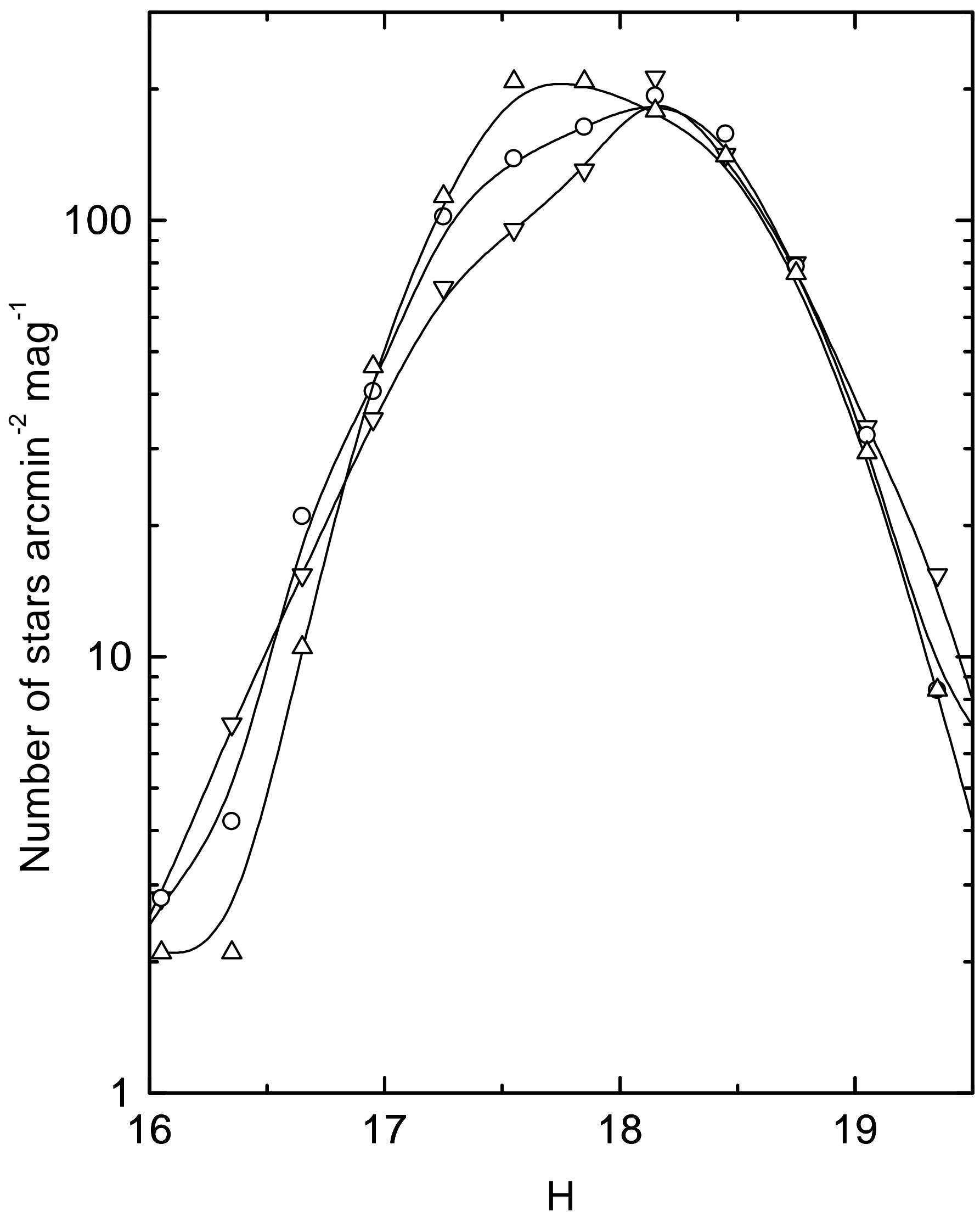




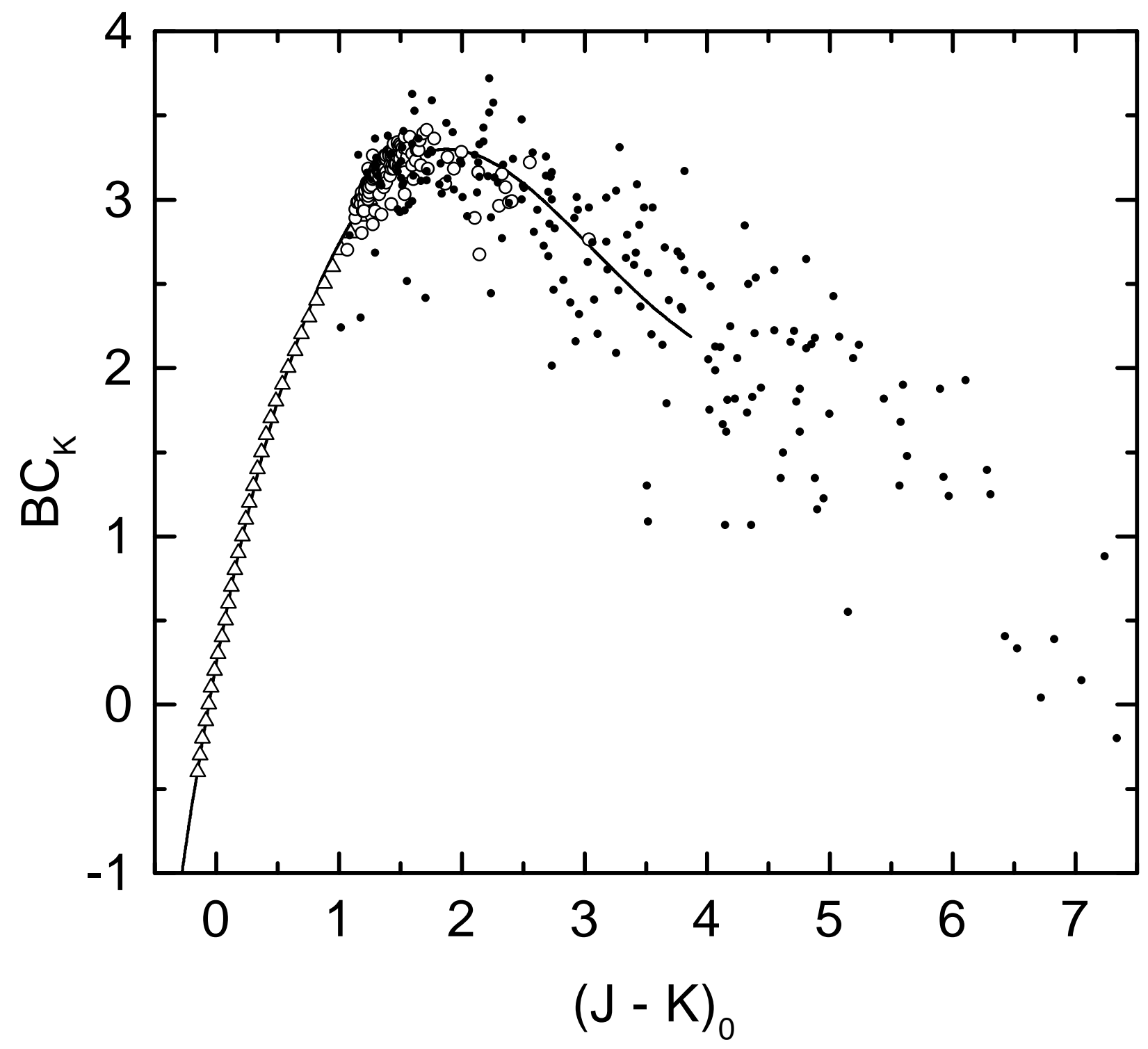

